\title{
Neurological Examination of the Upper Limb: A Study of Construct Validity
}

\author{
Jørgen R. Jepsen*,1, Lise H. Laursen ${ }^{1}$, Svend Kreiner ${ }^{2}$ and Anders I. Larsen ${ }^{3}$ \\ ${ }^{I}$ Department of Occupational Medicine, Sydvestjysk Sygehus Esbjerg, Østergade 81-83, DK-6700 Esbjerg, Denmark \\ ${ }^{2}$ Department of Biostatistics, University of Copenhagen, DK-2200 Copenhagen, Denmark \\ ${ }^{3}$ Occupational Health Services, Novozymes, DK-2880 Bagsvard, Denmark
}

\begin{abstract}
Objective: We have previously demonstrated that neurological individual findings and patterns can be reliably assessed in the examination of the upper limb and also that they are related to pain, weakness, and/or numbness/tingling. This study aimed to study further aspects of the construct validity of the neurological examination.

Methods: Blinded to patient-characteristics, two examiners assessed the function of 16 muscles, the sensibility in 7 territories, and the nerve-mechanosensitivity at 20 locations in 82 upper limbs. Based on anatomical patterns and pre-designed algorithms, one or both examiners rated neuropathy as "possible" or "definite" in 40 limbs and also determined the location(s). We developed and tested hypotheses on anatomically and regionally related locations of nerve afflictions (a selective vulnerability of neurons, double and multiple crush, and a tendency to regional spread) and examined the stability of the internal structure of the constructs in different situations. The interrelations of findings were analyzed by hypothesis testing and factor analyses, and the homogeneity of location profiles was analyzed by a conditional likelihood test.

Results: Out of 30 limbs with related locations of neuropathy, the findings of each examiner correlated positively (gamma $>0.35$ ) in $22 / 25$, respectively. The patterns of the interrelations identified by the two examiners were similar, with no evidence of any heterogeneity of location profiles for either examiner.

Conclusions: This study supports the validity of the physical examination. However, feasibility of its application requires the demonstration of further aspects of construct validity and a favorable influence on patient-management and/or prevention.
\end{abstract}

Keywords: Upper limb physical examination, construct validity, hypothesis testing, factor analyses, conditional likelihood test.

\section{INTRODUCTION}

The management of work-related upper limb symptoms frequently causes frustrations because the majority of patients cannot be diagnostically classified according to current criteria [1]. These constraints influence the long-term prognosis which may be grave with symptoms and limitations persisting in $83 \%$ after a median follow- up of 12 years [2]. Thus there is a need for valid and feasible clinical diagnostic tests and for reaching consensus with regard to practical case-definitions.

A triad of symptoms consisting of pain, weakness, and/or numbness/tingling frequently characterizes patients that cannot be diagnostically classified by common criteria. These symptoms suggest the presence of a neuropathic condition. The application of a detailed neurological examination to a sample of patients in occupational medicine, some of whom symptomatic and others without upper limb symptoms, permitted the frequent identification of patterns of dysfunction reflecting the topography and innervation patterns of nerves. The reliable identification of these patterns [3], and the

*Address correspondence to this author at the Department of Occupational Medicine, Sydvestjysk Sygehus, Østergade 81-83, DK-6700 Esbjerg, Denmark; Tel: +45 79182285;

E-mail: Joergen.Riis.Jepsen@svs.regionsyddanmark.dk relation of patterns to the mentioned symptoms [4], suggest the construct validity of the examination. When this examination is being applied to patients in the primary health sector [5], occupationally exposed patients [6], and active workers [7] (exemplified by computer operators), it can frequently reveal patterns of neurological findings suggesting peripheral neuropathies with specific locations.

This study addresses further aspects of construct validity in a sub-sample of previously reported patients $[3,4,8]$. The overall objective is to determine whether the outcome of the applied neurological examination reflects constructs of neuropathy, i.e. whether individual neurological findings and neurological patterns are theoretically founded and empirically relevant in relation to phenomena such as the underlying pathology that has caused them.

\section{Validity - Concepts}

Construct validity is the broadest type of validity and may be supported by content and criterion-related validity [9]. At times, laboratory studies, including the measurement of nerve conduction velocity and magnetic resonance imaging may support the diagnosis of focal neuropathy. However, they cannot serve as gold standards for the physical examination. Firstly, these studies should be preceded by a prior detailed neurological examination aiming to precisely locate 
the pathology. Secondly, they can neither confirm nor exclude neuropathy with certainty. Therefore, we have previously compared neurological findings to the presence of symptoms [4]. This comparison, however, is imperfect since symptoms may be unrelated to the nerves. In addition, neuropathy may occur in the absence of symptoms [10]. Consequently, determination of the diagnostic role of the neurological examination requires studies of additional aspects of construct validity.

There is no single method to determine construct validity, which is rather assessed by accumulating evidence resulting from numerous studies. Basically, the construct validity of a diagnostic approach to a disorder demands 1) definition of the content domain that represents the construct; 2) definition according to a theoretical context of the constructs of the disorder and of the employed diagnostic test; and 3) statistical comparison of data relating to the pathophysiology of the disorder and to the test. Hypothesis testing" involves the definition and testing of specific hypotheses that support the theoretical basis of the applied test and the constructs included. "Latent structure analysis" (confirmatory factor analyses based on item-response models) examines the factor structure of a test by testing different populations to ensure the stability of the internal structure (the factors or constructs) in different diagnostic subgroups. Each factor represents a group of test items or behaviors related to each other, but not to other factors within the test; and each factor reflects a different theoretical component of the overall construct [9].

\section{Constructs of Neuropathy and of the Diagnostic Approach}

Validation of a diagnostic test demands the testing of specific hypotheses reflecting the knowledge base of the theoretical foundation of the disorder and the test, and of the theoretical constructs measured.

\section{Constructs of Neuropathy}

The content domain and the constructs of neuropathy according to a theoretical context consist of the anatomical and pathophysiological characteristics of the peripheral nerves including their reactions to lesions and repair, and the tendency of a focal nerve affliction to extend topographically beyond its initial location [11-15].

External compression, tightening, or traction of nerves, e.g. from extreme or protracted limb positions or forceful or repetitive exertions, may acutely or chronically elevate the tissue pressure and cause perineural irritation. This is particularly likely to happen at anatomical locations with narrow passages where the bulging of synovium or muscle may further reduce the available space. Consequent to the elevated pressure, vascular supply and drainage may be compromised. Neurogenic inflammation leading to edema may cause further elevation of the tissue pressure, fibrotic adhesion to the surrounding structures and reduced nerve mobility [11-14]. Prolonged static position or exertion may result in progressive muscular imbalance with shortened or hypertrophied agonist muscles and weakened antagonists [11]. These altered topographical circumstances may result in further reduction of the space available for the nerve and compromise its mobility and integrity.
Such unfavorable circumstances for the nerve trunk may eventually lead to demyelination and/or axonopathy. The impaired impulse propagation may manifest itself by paresis and/or altered sensory input [16-19]. The involvement of the nervi nervorum [20] causes pain. Phenomena such as ectopic electrogenesis [21] and central modulation [22] are other potential consequences of a peripheral nerve-affliction.

The vulnerability of neurons depends on factors such as fiber-type and size, the size of the fascicle and its epineurial embedding, the location of the fiber within the fascicle, and the subject's general state of health. In addition, neurons in fascicles located superficially in a nerve trunk are more at risk from external impairment than those in fascicles located deeply inside nerves, and segmental affliction is more harmful to neurons than uniform compression [23]. This selective vulnerability results in mixed and partial nerve-afflictions with the majority of the fast conducting myelinated fibers left intact. Consequently, the conduction velocity is generally measured in the normal range [24,25]. This is a characteristic feature of, e.g. median neuropathy at elbow level [26] and brachial plexopathy [27].

The demonstration by ultrasound [28] and magnetic resonance imaging [29] of reduced median nerve mobility in the carpal tunnel in patients with "non-specific" upper limb pain suggests that the entire length of nerve is involved in these patients. These findings may also explain the clinically and experimentally demonstrated phenomenon that focal neuropathy at a certain level of the nerve renders the nerve itself more vulnerable distally ("double crush") and proximally ("inverse double crush") [30-33]. "Multiple crush" may result from the simultaneous affliction of several nerve segments. Such progressive involvement of a nerve in response to successive or continuous adverse exposures may manifest itself clinically as further progression of disease.

Depending on the anatomical features, an affliction of one particular nerve is unlikely to occur independently of an affliction of another nerve at the same level. For example, compression of the median nerve related to a maximal forearm pronation (by active contraction of the pronator teres muscle) is likely to compromise the posterior interosseous nerve as well because of the passive stretching of the supinator muscle over the nerve-passage at the Arcade of Frohse. Such simultaneous involvement of several nerves ("multiple entrapment") [31,32] may explain the significant associations of pain at adjacent sites [34].

From these considerations, the pathology and functional disturbances with a focal upper limb nerve affliction may be characterized by certain features:

- $\quad$ The pathology is mainly located at anatomical locations with limited available space at which the nerve is particularly prone to compression, stretching, tethering, or adherence to surrounding structures with specific movements or postures.

- The pathology involves one or several specific nerve(s) and location(s) within their course.

- $\quad$ The pathology has a smaller or larger extension along the nerve(s) (as opposed to a location limited to a specific small point). 
- $\quad$ The pathology is likely to spread successively and eventually involve several and/or larger nervesegments.

\section{Constructs of the Diagnostic Approach}

Diagnostic clues at the physical examination include the presence of mechanical allodynia of nerve trunks at locations with neuropathy [35] and functional disturbances due to selective motor or sensory impairment [36]. Consequently, the construct validity of the upper-limb neurological examination can be illustrated by the extent to which it can be shown to measure a hypothetical construct by 1) qualitative data and 2) quantitative data. 1) The qualitative data shows whether identified deviations from normal represent disorders confined to or involving the peripheral nerves. 2) The quantitative data demonstrates whether the meaningfulness of the measure illustrates the issue of whether correlations that should be found according to theoretical considerations are, in fact, identified.

\section{Hypotheses}

According to the constructs of neuropathy and of the neurological examination, one would expect a statistical correlation between the identification of neuropathy at related, but not at unrelated locations. This can be studied by testing three hypotheses concerning the interrelations between classifications as neuropathy at specific locations by the applied physical approach (Hypotheses $1-3$ ) and a fourth hypothesis relating to the application of the physical approach in various clinical situations (Hypothesis 4):

1. Neurological patterns indicating neuropathy at locations along the same nerve or in the same topographical region are strongly correlated.

2. Correlations between neurological patterns indicating neuropathy at locations which cannot be characterized as "inter-connected" (vs. above), can be explained by reference to neuropathy at locations separated from these locations.
3. Correlations between physical findings indicating neuropathy at two "inter-connected" locations cannot be explained through the extent of presence of neuropathy located separated from these locations.

4. The internal structure of the constructs of the diagnostic approach is stable in different situations.

\section{MATERIAL AND METHODOLOGY}

\section{Patients}

83 consecutive patients were referred from general practitioners and specialists to a hospital department of occupational medicine. Patients could suffer from any disorder and were preferentially referred for an assessment of its potential work-relatedness, and of the consequences of the conditions for the ability to work. Many were referred due to diagnostic difficulties, no response to prior treatment, or the recurrence of symptoms on resuming work. We excluded 17 patients because they were known from earlier contacts or because they had problems concerning communication, had undergone previous upper limb surgery, or had an appearance suggesting easily recognizable disease such as severe asthma or disabling low back disease. Fifteen patients refused participation. For capacity reasons (max. one study patient/day), ten patients comparable to the study patients with respect to disease pattern and severity were additionally excluded. The resulting sample of 41 patients was identical to a sample described previously $[3,8]$.

Twenty-two patients were males of median age 44 (range 29-61) years and 19 were females of median age 39 (range 25-52) years. Twenty-two patients were referred due to complaints from one and five patients due to complaints in both upper limbs. Among patients referred for other potentially work-related disorders, six had present and three had prior complaints from one or both upper limbs, while five patients were without present or prior upper limb complaints.

The study was approved by the local Ethics Committee and signed informed consent obtained from all patients.

Table 1. Neurological Items Examined

\begin{tabular}{|c|c|}
\hline Individual Muscle Strength & Sensibility in Homonymous Territories \\
\hline $\begin{array}{l}\text { Posterior deltoid } \\
\text { Pectorals } \\
\text { Biceps brachii } \\
\text { Triceps } \\
\text { Short extensor of wrist } \\
\text { Radial flexor of wrist } \\
\text { Subscapular } \\
\text { Latissimus dorsi } \\
\text { Infraspinatus } \\
\text { Serratus anterior } \\
\text { Rhomboid } \\
\text { Long flexor of thumb } \\
\text { Abductor pollicis brevis } \\
\text { Long extensor of thumb } \\
\text { Deep flexor to } 5^{\text {th }} \text { finger } \\
\text { Abductor of small finger }\end{array}$ & $\begin{array}{l}\text { Axillary nerve (deltoid region) } \\
\text { Medial cutaneous of arm nerve (medial upper arm) } \\
\text { Medial cutaneous of forearm nerve (medial forearm) } \\
\text { Musculocutaneous nerve (dorsal forearm) } \\
\text { Radial nerve (1st dorsal web) } \\
\text { Median nerve (volar tip of index finger) } \\
\text { Ulnar nerve (volar tip of 5th finger) }\end{array}$ \\
\hline
\end{tabular}



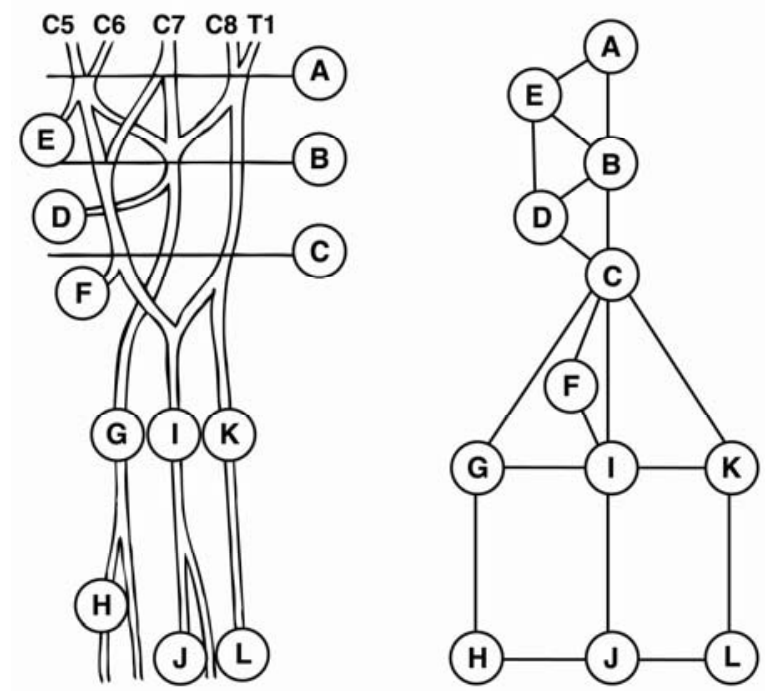

Fig. (1). Examined locations of neuropathy along the course of upper limb nerves.

Circles represent locations for neuropathy. A: Supraclavicular brachial plexus (scalene triangle), B: Clavicular brachial plexus, $\mathbf{C}$ : Infraclavicular brachial plexus (minor pectoral muscle), E: Suprascapular nerve (suprascapular notch), D: Axillary nerve (quadrilateral space), F: Musculocutaneous nerve (passage through coracobrachial muscle), G: Radial nerve (triceps arcade, brachioradial arcade, passage over radiohumeral joint), H: Radial nerve (arcade of Frohse), I: Median nerve (medial aspect of upper arm, lacertus fibrosus, pronator teres muscle, arcade of superficial flexor muscle), J: Median nerve (carpal tunnel), K: Ulnar nerve (arcade of Struther, medial head of triceps muscle, sulcus of ulnar nerve, aponeurosis of ulnar flexor of wrist), L: Ulnar nerve (canal of Guyon).

Lines represent the inter-correlations studied by hypothesis testing of conditional correlations.

\section{Methods}

One out of two primary examiners (both board certified specialists in occupational medicine) interviewed the participating patients about upper limb symptoms and performed a physical examination based on the protocol of ViikariJuntura [37]. A preliminary diagnostic statement was made concerning the presence, type and likely locations of diagnosed musculoskeletal disorders.

Independently of each other, in immediate succession one after the other, and blinded to any patient characteristics, two examiners performed identical physical examinations based on the classical neurologic examination [36] as previously described [3,8]: The voluntary isometric function was manually and semi-quantitatively studied in 16 individual upper limb muscles accessible for testing. Aesthesia (light touch) and algesia (pinprick) was assessed in seven homonymously innervated cutaneous areas and the vibratory sense by means of a $256 \mathrm{~Hz}$ tuning fork at the $2^{\text {nd }}$ and $5^{\text {th }}$ fingertip (Table 1). Mechanosensitivity of nerves was evaluated with a gentle manual pressure of $3 \mathrm{kp}$ on 20 potential locations along the nerves (Fig. 1). The physical items were rated according to Table 2.

Based on rating scales integrating the individual parameters, each limb was classified with regard to the presence of neuropathy as "definitely" present, "possibly" present, or "absent". The rating scales were arbitrarily designed to preferably exclude classification as "definite" or "possible" neuropathy. A number of neurological patterns were defined each of which reflected a specific location of neuropathy [3].

- For sensory nerves (the medial cutaneous nerves of arm and forearm and the radial nerve superficial branch) the classification was based on disturbances of aesthesia, algesia, and/or perception of vibration combined with nerve soreness. Neuropathy was classified as "definite" with a total score $\geq 4$ and as "possible" with a score of 2 or 3 and abnormal sensibility and soreness.

- $\quad$ For motor nerves (dorsal scapular, long thoracic, suprascapular, pectoral, thoracodorsal, subscapular, posterior and anterior interosseous nerves) the classification was based on reduced muscular strength combined with nerve soreness. Neuropathy was classified as "definite" with a total score $\geq 5$ and as "possible" with a score of 3 or 4 and abnormal strength and soreness.

- For mixed sensory/motor nerves (axillary, musculocutaneous, radial, median, and ulnar nerves), the classification was based on disturbances of aesthesia, algesia, and/or perception of vibration, and reduced

Table 2. Quantification of the Neurological Examination [36]

\begin{tabular}{|l|l|}
\hline $\begin{array}{l}\text { Manual testing of isometric strength in individual } \\
\text { muscles. } \\
\text { Quantification into five levels }\end{array}$ & $\begin{array}{l}\text { Grade 5: Contraction against powerful resistance, normal power }(\mathrm{score}=0) \\
\text { Grade 4+: Contraction against gravity and strong resistance }(\text { score }=1) \\
\text { Grade 4: Contraction against gravity and moderate resistance }(\text { score }=2) \\
\text { Grade 4-: Contraction against gravity and slight resistance }(\text { score }=3) \\
\text { Grade 3: Contraction against gravity }(\text { score }=3) \\
\text { No pareses minor than grade 3 were observed }\end{array}$ \\
\hline $\begin{array}{l}\text { Sensibility to light touch, pain (pinprick), and } \\
\text { vibration (tuning fork 256 Hz). } \\
\text { Quantification into three levels }\end{array}$ & $\begin{array}{l}\text { Normal (score }=0) \\
\text { Mild/any deviation of sensibility }(\text { score }=1)\end{array}$ \\
\hline $\begin{array}{l}\text { Nerve trunk mechanosensitivity assessed by palpa- } \\
\text { tion. } \\
\text { Quantification into four levels }\end{array}$ & $\begin{array}{l}\text { No/normal tenderness (score }=0) \\
\text { Mild/any mechanical allodynia }(\text { score }=1)\end{array}$ \\
\hline
\end{tabular}


Table 3. Locations of Neuropathy in 40 Limbs

\begin{tabular}{|c|c|c|c|}
\hline Location & $\begin{array}{c}\text { Identified } \\
\text { by Both } \\
\text { Examiners }\end{array}$ & $\begin{array}{c}\text { Identified } \\
\text { by } \\
\text { Examiner 1 }\end{array}$ & $\begin{array}{c}\text { Identified } \\
\text { by } \\
\text { Examiner }\end{array}$ \\
\hline Brachial plexus & 8 & & 1 \\
\hline Brachial plexus and branch(es)* & 3 & & 1 \\
\hline Brachial plexus, branch(es)*, radial nerve & 5 & & \\
\hline Brachial plexus, branch(es)*, radial, median, ulnar nerves & 2 & & 1 \\
\hline Branch(es)* & 2 & & \\
\hline Brachial plexus and radial nerve & 1 & & \\
\hline Brachial plexus, radial and median nerves & 1 & & \\
\hline
\end{tabular}

*Axillary, Suprascapular, or Musculocutaneous Nerves.

muscular strength in combination with nerve soreness. Neuropathy was classified as "definite" with a total score $\geq 6$ and as "possible" with a score of 4 or 5 and abnormal strength and soreness.

The algorithm above permitted the identification of the most proximally located neuropathy along a nerve. With neurological deficits compatible with an affliction of both the proximal and the distal course of a nerve, a distal nerve affliction was additionally diagnosed when, according to the rating scales (Table 2 ), the combined scores reflecting the distal deviation from normal of muscle strength, sensibility, and/or mechanosensitivity, were at least as high as the combined scores reflecting the proximal deviations from normal. Limbs classified with neuropathy at more than one location were recoded with all final locations for neuropathy illustrated in Table $\mathbf{3}$.

\section{Final Sample Subjected to Statistical Analyses}

Out of the 82 upper limbs of 41 participating patients, "definite" or "possible" neuropathy was identified by one of the blinded examiners in 34 limbs, and by the other in 36 limbs with agreement in 30 limbs [3]. The statistical analyses were applied for 40 limbs classified as "definite" or "possible" neuropathy by one or both examiners.

\section{Statistics}

The statistical considerations concerning the testing of the hypotheses 1. -3 . are based on the following: " 1 " may result from a tendency to the spread of neuropathy in the same nerve or topographical region. " 2 " is the consequence of the fact that neuropathy can propagate from one location to another only through nerve-segments located in between the two locations or due to shared topographic precipitating factors. "3" demands a positive partial correlation between neuropathies located close to each other (e.g. within the same nerve or in the same anatomical area). Consequently, the extension of neuropathy from one location to another should not be explained through the presence of neuropathy at nonintervening locations. "4" is hypothesized because the neuro- logical examination should remain applicable with various expressions of the disorder, e.g. in terms of severity and location.

Provided that the constructs are plausible and based on realistic considerations, and that it is reasonable to diagnose neuropathy through the neurological examination, identified positive correlations should be due to the defined constructs and should not be explained through control for other variables.

If the studied locations of neuropathy represent a complete set of potential locations, the partial correlation between the definitions of neuropathy at two distant locations would be 0 . The partial correlation is a conditional correlation coefficient, in which the correlation between two variables is assumed to be influenced by the value of one or several other variables. In this case, the influence of neuropathy defined at intermediate locations should be controlled for, for example the conditional independence of supraclavicular brachial plexopathy with ulnar neuropathy in the canal of Guyon, taking into account the physical indications of neuropathy along the intermediate portions of the ulnar nerve. If, however, the options for locations of neuropathy in the study do not represent all potential locations, positive correlations may occur. This, in fact, was the case in the presented study in which we selected a limited number for further studies. The 12 potential locations of neuropathy in Fig. (1) were regarded as of most clinical importance. The graphical models in Fig. (2) represent a redefinition of Fig. (1) with each circle representing one variable = location of neuropathy. According to the graphical model and the considerations above, the hypotheses of conditional independence between two neighboring variables given other variables in the model should be rejected. Due to insufficient data for testing such hypotheses, the testing was limited to hypotheses concerning conditional dependence between two neighboring variables given so-called separators, i.e. variables that would separate two variables in the graph in case of conditional independence [38]. Testing was performed individually for each examiner. 
a

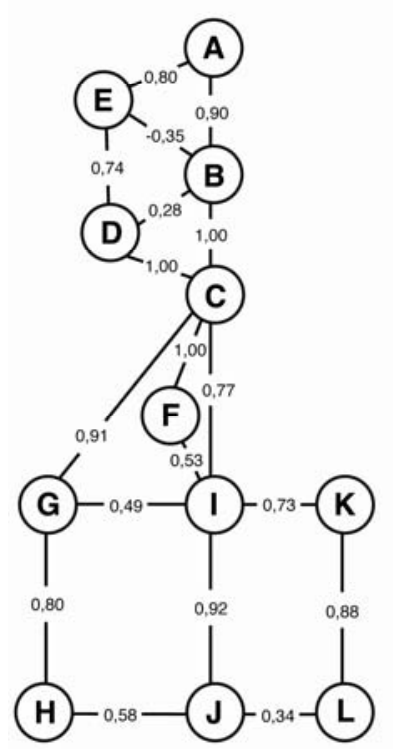

b

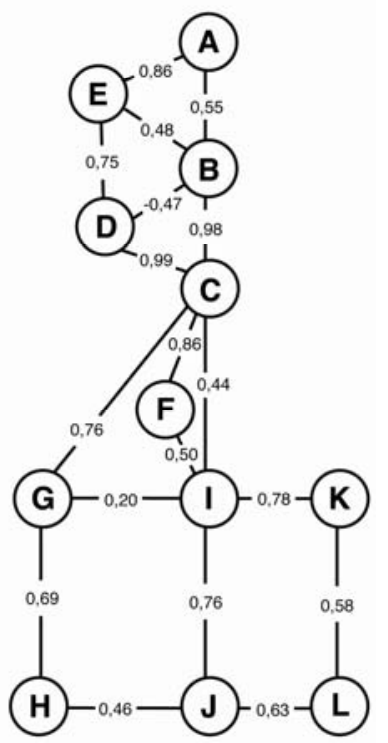

Fig. (2). Statistical correlations for examiner 1 (a) and 2 (b). The circles represent locations of neuropathy (Legends for locations in Fig. 1). Lines represent the inter-relations studied by hypothesis testing of conditional correlations. The coefficient of partial correlation is given for each of the studied inter-relations (gamma > 0.35 expressing a strong correlation).

Latent structure analyses of the stability of the constructs in different situations were based on Rasch analyses (conditional likelihood ratio tests) [39]. These analyses consider the underlying construct validity by assuming that the phenomenon of neuropathy is characterized by location profiles reflecting the relative susceptibilities of nerves at different locations. For the neurological examination to be valid, the severity of neuropathy in specific limbs should be independent of any other variables, including score-group status in the rating scales, independent of anamnestic information about symptom status and the side of the affected limb, and independent of the diagnostic conclusions of the primary investigators [4]. Assuming such independence, the Rasch model describes the risk of defining neuropathy at specific locations as a logistic function of a parameter relating to a limb indicating the severity of neuropathy for this specific limb and a location parameter describing the relative susceptibility at the location in question (location profile). The model implies that we regard the total number of locations with neuropathy in this study as an objective measurement of neuropathy that represents a sufficient statistical measure for its severity, and that the location profiles estimated in different subgroups should not differ significantly.

\section{RESULTS}

\section{The Findings of the Examiners}

Out of 40 limbs rated by any of the two blinded examiners as "definite"/"possible" neuropathy, 22 and 16 limbs, respectively, were classified as "definite", and 12 and 20 limbs, respectively, as "possible" neuropathy. Dichotomous agreement ("definite"/"possible" neuropathy vs. "absence" of neuropathy) was achieved in 22 right and 8 left limbs $(87.8 \%)[3]$.
The disagreement between the two examiners increased with the subdivision of locations of neuropathy, e.g. various levels within the brachial plexus or the median nerve. A higher agreement was reached with combinations of involved locations, e.g. of the brachial plexus per se or of some peripheral nerve. Based on the defined patterns [3], neuropathy was mostly identified in the brachial plexus and largely with the additional involvement of the median and/or radial/posterior interosseous nerves at elbow level, conceivably as "double crush" phenomena (Table 3).

\section{Correlation Between the Findings of the Two Examiners}

For each examiner we have calculated the direct correlations between two individual locations of neuropathy that cannot be explained through correlations with neuropathy assigned to other locations. For both examiners the connecting lines in the graphical models in Fig. (2) illustrate the statistically significant direct positive correlations between the locations of neuropathy that are not interconnected through another location. Table 4 illustrates all resulting calculations of the coefficient of partial correlation expressed by the gamma-coefficient (with gamma $>0.35$ expressing a strong correlation). Positive partial correlations between individual anatomically and regionally related locations of neuropathy were present in $22 / 25$, respectively, out of 30 interrelationships. In between the two examiners, the interrelations of various locations (patterns) of neuropathy were similar (Table 4, Fig. 2).

\section{Location Profiles in Different Subgroups}

In more than one case in the studied sample, focal neuropathy was defined at the supra- and infraclavicular brachial plexus, the suprascapular nerve, and the median nerve at elbow and wrist level. The analyses by the so-called conditional likelihood ratio test [39] of the homogeneity of the location profiles in different subgroups were therefore restricted to these locations. No evidence of heterogeneity was observed for either examiner (Table 5). This finding implicates the independence of the profile of selective vulnerability in relation to the score-group status according to the rating scales, the symptom status, the anamnestic conclusion of the primary investigators, and the involved side.

\section{DISCUSSION}

A clinical diagnosis based on the integration of historyderived symptoms and signs infer a mutual risk of the examiner being biased from either of these. Depending on the physical qualities examined, findings may be normal or deviate only slightly from normal in patients with upper limb complaints [15]. Still, a detailed and systematic testing of individual muscular strength, the mapping of sensibility, and the assessment of nerve trunk mechanosensitivity frequently permits the identification of neurological patterns in accordance with anatomy. These patterns can be reliably identified [3], and their presence correlates to symptoms that may be caused by peripheral nerve-dysfunction [4].

While the absence of an applicable "golden" standard criterion prevents the assessment of criterion-related validity of the neurological examination, the support of the hypotheses (Table 4) is an indication of construct validity. We do not regard the remarkable significance of most partial correlations as representing a coincidence, especially because of the 
Table 4. Hypothesis Testing of Conditional Correlation

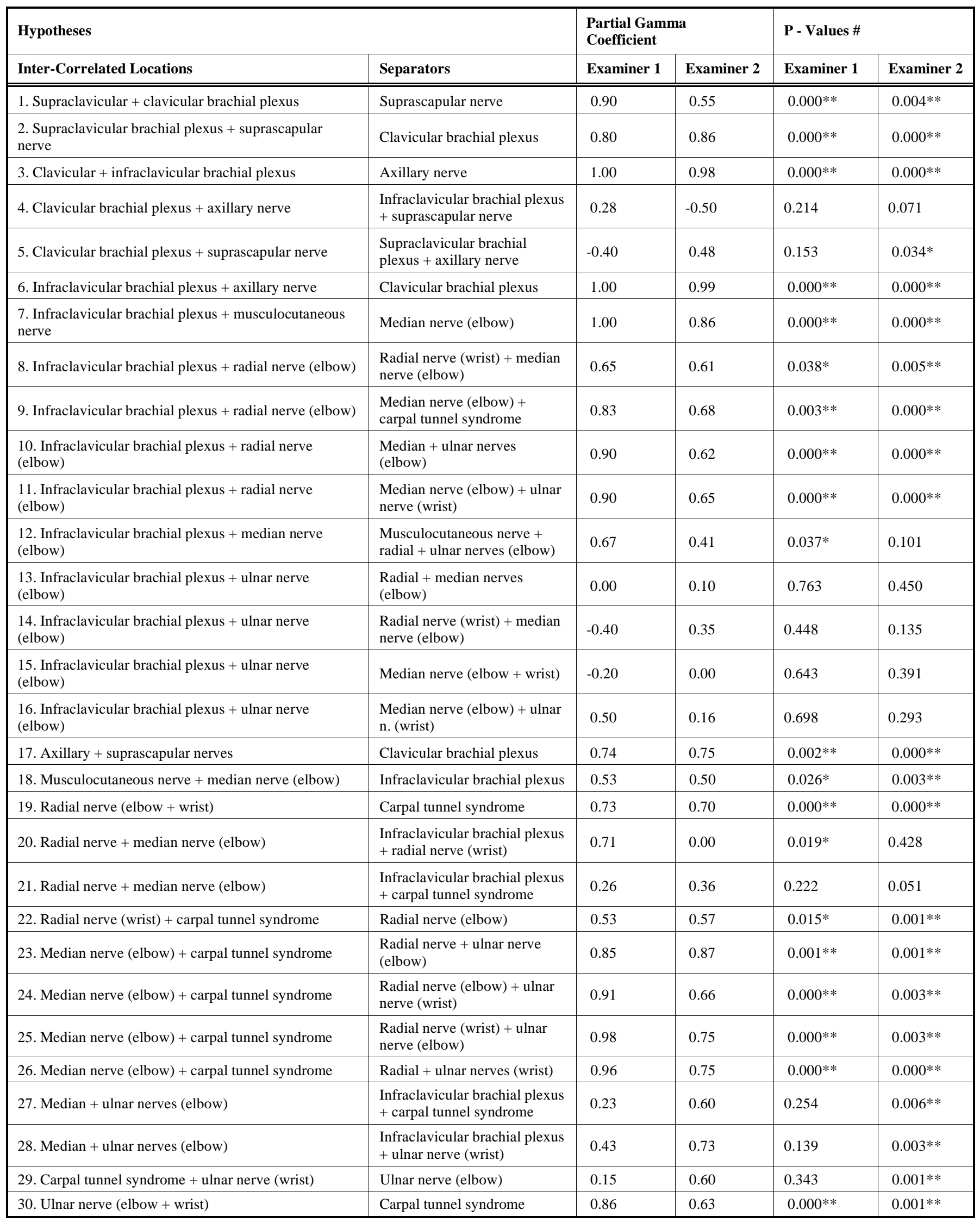

The partial correlation between two individual locations of neuropathy that cannot be explained through correlations with neuropathy assigned to other locations. The coefficient of correlation is expressed by the gamma-coefficient (gamma $>0.35$ expresses a strong correlation). \# Monte Carlo estimates of exact $\mathrm{P}$-values [40]. *P $<0.05$ (one-sided). **P< 0.01 (one-sided). 
Table 5. The Homogeneity of Location Profiles in Different Subgroups Studied by a Conditional Likelihood Ratio Test [39]. The Outcome for both Examiners was not Influenced by the Severity of the Disorder Expressed by Physical Findings or Symptoms, by the Previous Assessment of the Primary Investigators, or by the Disorder Being on the Left or Right Side

\begin{tabular}{|l|c|c|c|c|c|c|}
\hline \multirow{2}{*}{ Subgroups Defined } & \multicolumn{3}{|c|}{ Examiner 1 } & \multicolumn{2}{c|}{ Examiner 2 } \\
\cline { 2 - 7 } & $\mathbf{X}^{\mathbf{2}}$ & df & P & $\mathbf{X}^{2}$ & \multicolumn{1}{c|}{ Pf } & 5 \\
\hline \hline Score-group status & 6.2 & 5 & 0.29 & 3.8 & 5 & 0.31 \\
\hline Symptoms & 4.8 & 5 & 0.44 & 9.9 & 10 & 0.45 \\
\hline Anamnestic conclusion & 4.9 & 10 & 0.14 & 1.4 & 5 \\
\hline Side of affected limb & 4.8 & 5 & 0.45 & 0.93 \\
\hline
\end{tabular}

limited number of limbs and individual locations of neuropathy. This finding suggests that the examination may provide diagnostic assistance to the high number of upper limb patients that cannot be diagnosed with a standard physical approach [1].

However, for both raters there were a few exceptions to the general trend of significant partial correlations: The negative (non-significant) partial correlation for the presence of infraclavicular brachial plexopathy and ulnar neuropathy at elbow level could not be explained by median and radial neuropathy (hypotheses number 13 and 15). Negative or weak and mostly insignificant correlations were also noticeable for both examiners concerning a few other hypotheses (number 4 and 27, and for one examiner additionally number $5,14,16,21,27$ and 29). The few locations with negative or low correlations for one or both examiners may be explained from their infrequency in the sample, e.g. ulnar neuropathy was found in two limbs only (elbow and wrist levels, respectively) and carpal tunnel syndrome was discovered by one of the two examiners in one limb (Table 4).

The main weakness of this study is the potential bias that may be induced because all physical parameters were examined by the same two examiners. In spite of the blinding of the physical examination with regard to patient-related information, the examiners' findings may have been influenced by expectations. For example, it may be argued that the weakness in a specific muscle could distort the interpretation of nerve trunk soreness or sensory deviation from normal at specific locations anatomically relevant to the identification of weakness. We cannot allow for this potential bias due to the design of the study. If present, it would tend to improve the intra-examiner partial correlations of topographical clustering of neuropathy in accordance with anatomy, which was demonstrated for both examiners (Fig. 2). The striking similarity of the relative distributions for the two examiners suggests the inter-examiner findings to be also correlated and argues against a major influence of such bias.

The power of the tests of fit in the Rasch model should be regarded in relation to the sample size. Nevertheless, in this small-sample study, the latent structure analysis of locations did not disclose any evidence of heterogeneity whatsoever for either examiner. Indicating the relative susceptibilities of nerves at different locations, the location profile for patients with neuropathy at one or a few locations was similar to the profile for patients with more widespread neuropathy.
Based on semi-quantitative ratings, simple techniques, and minimal training [3], the examination identified patterns relating to the topography, function and integrity of the upper limb nerves including their course from the roots, through the plexus, via a number of divisions to the ultimate innervation of muscles, skin, and other structures. Two findings in this study may appear surprising:

- The high number in a sample of patients from a department of occupational medicine of cases diagnosed as neuropathy (agreement in 30 out of 82 examined limbs [3]) indicate the extent to which potentially work-related upper limb musculoskeletal disorders may be confined to or involve the upper limb nerves. This finding is supported by reports of others of nonnociceptive and potentially neuropathic pain conditions secondary to the compression, tightening, and traction of the upper limb nerves $[14,16,41,42]$ in a substantial proportion of patients with unclassifiable ("non-specific") refractory cervicobrachial pain conditions occurring in an occupational context $[11,15,26,43,44]$. Abnormalities suggesting neural dysfunction include elevated vibratory thresholds [43], pathologic upper limb tension tests [15], axonal flare responses [45], hyperalgesia [18], and allodynic responses to supra-threshold vibratory stimulation [43].

- In the majority of symptomatic limbs, with the defined criteria [3], the examination indicated an involvement of the brachial plexus with the most frequent location at cord level behind the minor pectoral muscle. With this location an accompanying distal neuropathy was additionally identified in most limbs, in particular involving the median or/and radial/interosseous nerve - perceivable as a "double crush" phenomenon. The frequent occurrence of brachial plexopathy is controversial but supported by clinical experiences and pathophysiological reasoning [11]. Positive responses to the outward abduction test and Roos' test indicating a brachial plexopathy have been identified in a significant number of symptomatic workers $[46,47]$ with variable prevalence between occupational subgroups. Although these tests should not be considered diagnostic, it has been demonstrated that they can predict future upper limb pain and signs of neuropathy [48].

Upper limb focal neuropathy may cause non-specific symptoms located distant to the pathology. Consequently, it 
would be reasonable to demand that the neurological examination should extend beyond the symptomatic area and include the whole limb and neck. Otherwise a neuropathic condition, e.g. a brachial plexopathy, may be misinterpreted as a disorder in the symptomatic region or labeled as a "nonspecific" disorder.

While the relation to adverse exposures at work of most locations of upper limb neuropathy remains to be demonstrated, we are still at a stage where hypotheses for causal inference are created by the exposure-history and by pathophysiological reasoning [11]. These hypotheses, and the effect of subsequent control of ergonomic and organizational risk factors at work, should be tested in future epidemiological research based on well-defined effect measures, e.g. valid diagnostic criteria. The diagnosis also constitutes a basis for the treatment and rehabilitation of upper limb disorders. However, the current limitations in the management of common upper limb pain conditions including wrist tenosynovitis, lateral epicondylitis, rotator cuff syndrome, and myofascial pain syndrome [49] remain hampered from poorly defined clinical diagnostic criteria and tests with insufficient reliability and validity [50]. In spite of recent steps to define and validate case definitions [51,52], the conventional physical approach appears to be insufficient for diagnosing disorders which may be caused by a nerve affliction $[5,11,15,43,53]$. The application of the suggested neurological examination may contribute to a better diagnostic coverage and accuracy.

\section{CONCLUSION}

Previously, we have demonstrated the diagnostic precision of the studied semi-quantitative neurological upper limb examination with regard to individual findings and patterns $[3,8]$. In addition, we have shown that patterns suggesting focal neuropathy are more likely present in symptomatic subjects and absent in non-symptomatic subjects [4]. This study has provided further evidence of the construct validity of the neurological examination, and it suggests that it is possible to reduce the fraction of non-classifiable upper limb conditions by addressing the nerves in a systematic fashion (individual function of muscles, sensory function in homonymous territories, nerve trunk soreness). It remains to be shown in samples with various prevalence and severity of upper limb disorder that such an approach can lead to improved management or prevention. However, feasibility is suggested by the identification of similar neurological patterns in samples of patients in general practice [5], in patients with computer-related upper limb disorders [6], and among active symptomatic computer operators [7]. The demonstration of a favourable influence of an intervention targeting the neurological findings of the examination [54] may also indicate the value of a wider physical approach in the diagnosis of work-related upper limb disorders.

\section{ACKNOWLEGDEMENTS}

Financial support for this study was provided in part by a grant from Statens Sundhedsvidenskabelige Forskningsråd, Copenhagen, Den Samfundsvidenskabelige Forskningsfond, Ringkøbing, and Lida \& Oskar Nielsens Fond, Esbjerg. The funding agreement ensured the authors' independence in designing the study, interpreting the data, writing, and publishing the report.

\section{REFERENCES}

[1] Palmer K, Cooper C. In: Baxter P, Adams P, Aw T, Cockcroft A, Harrington J, Eds. Hunter's diseases of occupations. London: Arnold. 2000; pp. 453-475.

[2] Stone J, Sharpe M, Rothwell PM, Warlow CP. The 12 year prognosis of unilateral functional weakness and sensory disturbance. J Neurol Neurosurg Psychiatry 2003; 74: 591-6.

[3] Jepsen JR, Laursen LH, Hagert C-G, Kreiner S, Larsen AI. Diagnostic accuracy of the neurological upper limb examination I: interrater reproducibility of findings and patterns. BMC Neurol 2006; 6: 8.

[4] Jepsen JR, Laursen LH, Hagert C-G, Kreiner S, Larsen AI. Diagnostic accuracy of the neurological upper limb examination II: the relation to symptoms of patterns of findings. BMC Neurol 2006; 6: 10.

[5] Laursen LH, Sjogaard G, Hagert CG, Jepsen JR. Diagnostic distribution of non-traumatic upper limb disorders: vibrotactile sense in the evaluation of structured examination for optimal diagnostic criteria. Med Lav 2007; 98: 127-44.

[6] Jepsen JR. Upper limb neuropathy in computer operators? A clinical case study of 21 patients. BMC Musculoskeletal Disord 2004; 5: 26.

[7] Jepsen JR, Thomsen G. A cross-sectional study of the relation between symptoms and physical findings in computer operators. BMC Neurol 2006; 6: 40.

[8] Jepsen J, Laursen L, Larsen A, Hagert CG. Manual strength testing in 14 upper limb muscles: a study of the inter-rater reliability. Acta Orthop Scand 2004; 75: 442-8.

[9] Portney LG, Watkins MP. Foundations of clinical research: applications to practice. Upper Saddle River, NJ: Prentice Hall Health 2000.

[10] Neary D, Ochoa J, Gilliatt RW. Sub-clinical entrapment neuropathy in man. J Neurol Sci 1975; 24: 283-98.

[11] Novak CB, Mackinnon SE. Multilevel nerve compression and muscle imbalance in work-related neuromuscular disorders. Am J Ind Med 2002; 41: 343-52.

[12] Lundborg G. Nerve injury and repair: regeneration, reconstruction, and cortical remodeling. Philadelphia: Elsevier Churchill Livingstone 2005.

[13] Szabo RM, Gelberman RH. The pathophysiology of nerve entrapment syndromes. J Hand Surg Am 1987; 12: 880-4.

[14] Rempel D, Dahlin L, Lundborg G. Pathophysiology of nerve compression syndromes: response of peripheral nerves to loading. J Bone Joint Surg Am 1999; 81: 1600-10.

[15] Elvey RL, Quintner JL, Thomas AN. A clinical study of RSI. Aust Fam Physician 1986; 15: 1314-22.

[16] Kramis RC, Roberts WJ, Gillette RG. Non-nociceptive aspects of persistent musculoskeletal pain. J Orthop Sports Phys Ther 1996; 24: 255-67.

[17] Roberts WJ, Kramis RC. In: Fields HL, Ed. Pain syndromes in neurology. Oxford: Butterworths Heinemann 1990; pp. 85-106.

[18] Arroyo JF, Cohen ML. Unusual responses to electrocutaneous stimulation in refractory cervicobrachial pain: clues to a neuropathic genesis. Clin Exp Rheumatol 1992; 10: 475-82.

[19] Cohen ML, Arroyo JF, Champion GD, Brown CD. In search of the pathogenesis of refractory cervicobrachial pain syndrome: a deconstruction of the RSI phenomenon. Med J Aust 1992; 156: 432-6.

[20] Chen DL, Novak CB, Mackinnon SE, Weisenborn SA. Pain responses in patients with upper-extremity disorders. J Hand Surg Am 1998; 23: 70-5.

[21] Devor M, Rappaport ZH. In: Fields HL, Ed. Pain syndromes in neurology. Oxford: Butterworth Heinemann 1990; pp. 47-83.

[22] Gracely RH, Lynch SA, Bennett GJ. Painful neuropathy: altered central processing maintained dynamically by peripheral input. Pain 1992; 51: 175-94.

[23] Lundborg G, Dahlin LB. The pathophysiology of nerve compression. Hand Clin 1992; 8: 215-27.

[24] Dellon AL. In: Gelberman RH, Ed. Operative nerve repair and reconstruction. Philadelphia: Lippingcott, JB, Co. 1991; pp. 18596.

[25] Homan MM, Franzblau A, Werner RA, et al. Agreement between symptom surveys, physical examination procedures and electrodiagnostic findings for the carpal tunnel syndrome. Scand J Work Environ Health 1999; 25: 115-24. 
[26] Stål M, Hagert C-G, Moritz U. Upper extremity nerve involvement in Swedish female machine milkers. Am J Ind Med 1998; 33: 5519.

[27] Komanetsky RM, Novak CB, Mackinnon SE, et al. Somatosensory evoked potentials fail to diagnose thoracic outlet syndrome. J Hand Surg Am 1996; 21: 662-6.

[28] Greening J, Lynn B, Leary R, et al. The use of ultrasound imaging to demonstrate reduced movement of the median nerve during wrist flexion in patients with non-specific arm pain. J Hand Surg 2001; 26B: 401-6.

[29] Greening J, Smart S, Leary R, et al. Reduced movement of median nerve in carpal tunnel during wrist flexion in patients with nonspecific arm pain: a magnetic resonance imaging study. Lancet 1999; 354: 217-8

[30] Upton AR, McComas AJ. The double crush in nerve entrapment syndromes. Lancet 1973; 2: 359-62.

[31] Mackinnon SE, Dellon AL. Surgery of the peripheral nerve. New York: Thieme Medical 1988; pp. 360-368.

[32] Simpson RL, Fern SA. Multiple compression neuropathies and the double crush syndrome. Orthop Clin North Am 1996; 27: 381-8.

[33] Dellon AL, Mackinnon SE. Chronic nerve compression model for the double crush hypothesis. Ann Plast Surg 1991; 26: 259-64.

[34] Walker-Bone K, Reading I, Coggon D, Cooper C, Palmer KT. The anatomical pattern and determinants of pain in the neck and upper limbs: an epidemiological study. Pain 2004; 109: 45-51.

[35] Hall TM, Elvey RL. Nerve trunk pain: physical diagnosis and treatment. Man Ther 1999; 4: 63-73.

[36] The Editorial Committee for the Guarantors of Brain. Aids to the examination of the peripheral nervous system. London: Ballière Tindall 1986

[37] Viikari-Juntura E. Neck and upper limb disorders among slaughterhouse workers: an epidemiological and clinical study. Scand J Work Environ Health 1983; 9: 283-90.

[38] Blettner M, Sauerbrei W, Schlehofer B, Scheuchenpflug T, Friedenreich C. Traditional reviews, meta-analyses and pooled analyses in epidemiology. Int J Epidemiol 1999; 28: 1-9.

[39] Andersen EB. A goodness of fit test for the Rasch model. Psychometrika 1973; 38: 123-40.

[40] Kreiner S. Analysis of multidimensional contingency tables by exact conditional tests: Techniques and strategies. Scand J Stat 1987; 14: 97-112.
[41] Armstrong TJ, Buckle P, Fine L, et al. A conceptual model for work-related neck and upper limb musculoskeletal disorders. Scand J Work Environ Health 1993; 19: 73-84.

[42] Greening J, Lynn B. Minor peripheral nerve injuries: an underestimated source of pain. Man Ther 1998; 3: 187-94.

[43] Greening J, Lynn B. Vibration sense in the upper limb in patients with repetitive strain injury and a group of at-risk office workers. Int Arch Occup Environ Health 1998; 71: 29-34.

[44] Doezie AM, Freehill AK, Novak CB, Dale AM, Mackinnon SE Evaluation of cutaneous vibration thresholds in medical transcriptionists. J Hand Surg Am 1997; 22: 867-72.

[45] Helme RD, LeVasseur SA, Gibson SJ. RSI revisited: evidence for psychological and physiological differences from an age, sex and occupation matched control group. Aust N Z J Med 1992; 22: 23-9.

[46] Sällström J, Schmidt H. Cervicobrachial disorders in certain occupations, with special reference to compression in the thoracic outlet. Am J Ind Med 1984; 6: 45-52.

[47] Toomingas A, Hagberg M, Jorulf L, et al. Outcome of the abduction external rotation test among manual and office workers. Am J Ind Med 1991; 19: 215-27.

[48] Toomingas A, Nilsson T, Hagberg M, Lundström R. Predictive aspects of the abduction external rotation test among male industrial and office workers. Am J Ind Med 1999; 35: 32-42

[49] Nørregaard J, Jacobsen S, Kristensen JH. A narrative review on classification of pain conditions of the upper extremities. Scand J Rehabil Med 1999; 31: 153-64.

[50] Marx RG, Bombardier C, Wright JG. What do we know about the reliability and validity of physical examination tests used to examine the upper extremity? J Hand Surg Am 1999; 24: 185-93.

[51] Harrington JM, Carter JT, Birrell L, Gompertz D. Surveillance case definitions for work related upper limb pain syndromes. Occup Environ Med 1998; 55: 264-71

[52] Palmer K, Walker-Bone K, Linaker C, et al. The Southampton examination schedule for the diagnosis of musculoskeletal disorders of the upper limb. Ann Rheum Dis 2000; 59: 5-11.

[53] Mitchell S, Cooper C, Martyn C, Coggon D. Sensory neural processing in work-related upper limb disorders. Occup Med Lond 2000; 50: 30-2.

[54] Jepsen JR, Thomsen G. Prevention of upper limb symptoms and signs of nerve afflictions in computer operators: the effect of intervention by stretching. J Occup Med Toxicol 2008; 3: 1 .

Received: March 06, 2009

Revised: April 28, 2009

Accepted: June 03, 2009

(C) Jørgen Riis Jepsen et al.; Licensee Bentham Open.

This is an open access article licensed under the terms of the Creative Commons Attribution Non-Commercial License (http://creativecommons.org/licenses/by-nc/3.0/) which permits unrestricted, non-commercial use, distribution and reproduction in any medium, provided the work is properly cited. 